in sciences other than anatomy, biochemistry, physiology and pharmacology. On clinical placement, there is little crossspeciality support for those students who want to be psychiatrists and sometimes even lost opportunities for those publically aspiring towards psychiatry. Placements in psychiatry give students a better understanding of psychiatry but this does not seem to significantly change their career aspirations and this is rather defined from the admission stages.

Conclusion. After comparing experience with literature, stigma towards psychiatry appears to be universal. It may be important to consider the types of students who are being attracted to medical school as currently students seem to have an intrinsic disinterest in psychiatry despite later becoming better informed through psychiatric placement. Culture is notoriously hard to change, particularly within medicine. This stigma exists both in the lay and medical communities with early potentially inaccurate lay views of psychiatry being validated and reinforced throughout medical school.

Improving quality of psychiatry training in Northern Ireland through the introduction of postgraduate education fellows

Catherine Boucher ${ }^{1 \star}$, Roisin Connolly ${ }^{2}$, Michael Doris ${ }^{3}$, Colin Gorman ${ }^{4}$, Michael McMorran ${ }^{4}$ and Aisling Sheridan ${ }^{5}$

${ }^{1}$ South Eastern Health \& Social Care Trust; ${ }^{2}$ Southern Health \& Social Care Trust; ${ }^{3}$ Belfast Health \& Social Care Trust; ${ }^{4}$ Northern Health \& Social Care Trust and ${ }^{5}$ Western Health \& Social Care Trust ${ }^{\star}$ Corresponding author.

doi: 10.1192/bjo.2021.366

Aims. To improve postgraduate psychiatry education and training in Northern Ireland.

Background. Historically within Northern Ireland there has been a postgraduate Member of the Royal College of Psychiatrists (MRCPsych) teaching programme delivered to core trainees in preparation for MRCPsych examinations. There has been no official teaching programme for higher trainees. Northern Ireland Medical and Dental Training Agency (NIMDTA), in collaboration with the Royal College of Psychiatrists in Northern Ireland and all five Trusts developed the novel idea of introducing Postgraduate Education Fellows, to oversee and improve core training, and to develop a bespoke higher training programme.

The Postgraduate Education Fellows met to collate information from various sources in relation to issues within the current teaching programme and address these along with the development of new initiatives. The fellows further act as a point of contact for all trainees within their Trust to provide advice and support with education if needed.

Method. One higher trainee was appointed to the role of Postgraduate Education Fellow in each Trust within the NIMDTA deanery for a term of 1 year.

The starting point was delivering the pre-established teaching timetable and gaining feedback from core trainees to identify areas for improvement. The next phase involved piloting traditional and contemporary methods of feedback. A further development was designing a mock paper A delivered under exam conditions. Two mock Clinical Assessment of Skills and Competencies (CASC) exams were organised under exam conditions, offering other trainees the opportunity to act as simulated patients and examiners.

The third aspect of this role involved creating a programme of higher trainee seminars. Baseline data were collated and identified key areas that higher trainees felt they needed further training and guidance in.
Result. Using baseline data on the current teaching programme and from higher trainees as well as incorporating quality improvement methodology, we have been making small changes to each aspect of the teaching programme and evaluating the changes made. The feedback from trainees has been positive as evidenced by quantitative and qualitative feedback. 8 candidates sat our first mock CASC with a $100 \%$ pass rate in their MRCPsych CASC examination. There has been a positive response to the higher trainee seminar programme. Conclusion. This programme has produced good outcomes to date and sets foundations for the future development of post graduate psychiatry education in Northern Ireland.

\section{Virtual balint group experience due to the COVID-19 pandemic}

\author{
Rebecca Brown ${ }^{\star}$ and Nicola Philips \\ Nottinghamshire Healthcare NHS Foundation Trust \\ ${ }^{*}$ Corresponding author.
}

doi: 10.1192/bjo.2021.367

Aims. In the changes brought about by remote working, the local psychotherapy case discussion group (Balint Group) has developed as a remote service via video consultation. It is important to consider the effect that this change in method of delivery has had on experience.

Method. An anonymous survey was distributed to determine the benefits and challenges from participants and facilitators with at least a month of virtual Balint Group experience. The open-ended survey questions captured extended answer responses from 16 students and trainees, and 5 (co-)facilitators, within Nottinghamshire Healthcare NHS Foundation Trust. The qualitative feedback was analysed by thematic analysis, identifying three main themes.

Result. The first theme of practicalities was centred around access to the group. The virtual format had benefits in terms of reducing travel and time commitment and so improving attendance. However, disadvantages were in technological issues and finding a private and safe environment, individuals often not leaving the work environment on which they were reflecting.

The second theme of communication identified how virtual methods are a less natural way of interacting (for example sequential point making), losing both immediacy of reactions and nonverbal communication. There was a loss of essential communication cues, with disjointed conversation affecting contribution.

The third theme of group dynamics had some advantages, feeling less intimidating virtually. Yet disadvantages included loss of group cohesion, with participants not building the same relationships (on arriving and leaving a group space), and trust. The more subtle emotions in the group might be missed and opinions given less openly. The facilitators needed to be more directive and experienced difficulties maintaining group engagement and managing the frame.

Conclusion. The advantages of virtual format are more based on accessibility and the disadvantages more experiential. There are elements of being physically remote that lead to a disembodied experience, that might impact on capacity to reflect emotionally. This might make it more difficult to identify unconscious processes and the experience might be more cognitive. There is a risk that virtually participants will feel more alone with difficult feelings and unsupported by the group.

When mental health is being affected by social isolation due to the pandemic, having groups virtually can mimic this isolation in working life. Overall the preference remained for an in-person group. 